\title{
Tabaquismo en las enfermeras de un hospital nacional de Lima, Perú
}

\author{
Vilma Pérez Saavedra ${ }^{1}$ \\ Paulo Sérgio Ferreira ${ }^{2}$ \\ Sandra Cristina Pillon ${ }^{3}$
}

Las enfermeras poseen oportunidades únicas para ayudar a sus pacientes a dejar de fumar. El objetivo de esta investigación fue identificar el consumo, las actitudes y los conocimientos recibidos sobre el tabaco por las enfermeras de un hospital en Lima. Se trata de un estudio descriptivo realizado en 204 (48\%) enfermeras asistenciales de un Hospital Nacional en Lima, Perú, utilizando la Encuesta Mundial de Profesionales de la Salud (GHPS). Se identificó que la prevalencia de vida de uso de tabaco fue $67,1 \%$ y el último mes fue de 3\%; la mayoría de las enfermeras presentó actitudes positivas para el aspecto - dejar de fumar - y reconocieron la importancia y la responsabilidad de aconsejar a los pacientes a dejar ese hábito; las enfermeras recibieron conocimientos generales sobre el tema, pero la mitad no recibió conocimientos específicos sobre el tratamiento. Se concluye que frente a la importancia del tema se identificó que existe la necesidad de realizar más estudios de esta naturaleza, así como revisar los contenidos curriculares abordados acerca del tema tabaquismo.

Descriptores: Tabaquismo; Personal de Enfermería en Hospital; Enfermería.

\footnotetext{
${ }^{1}$ Licenciada en Enfermería, Maestra em Enfermería, Profesor, Facultad de Enfermería, Universidad Peruana Cayetano Heredia, Peru. E-mail: vperez@upch.edu.pe.

${ }^{2}$ Enfermero, Escola de Enfermagem de Ribeirão Preto, Universidade de São Paulo, Centro Colaborador de la OMS para el Desarrollo de la Investigación en Enfermería, Brasil. E-mail: pausefer@eerp.usp.br.

${ }^{3}$ Enfermera, Doctor, Profesor Doctor, Escola de Enfermagem de Ribeirão Preto, Universidade de São Paulo, Centro Colaborador de la OMS para el Desarrollo de la Investigación en Enfermería, Brasil. E-mail: pillon@eerp.usp.br.
}

Correspondencia:

Sandra Cristina Pillon

Universidade de São Paulo. Escola de Enfermagem de Ribeirão Preto

Av. Bandeirantes, 3900

Bairro Monte Alegre

CEP: 14040-902 Ribeirão Preto, SP, Brasil

E-mail: pillon@eerp.usp.br 


\section{Tabagismo entre enfermeiras do hospital nacional de Lima, Peru}

Os enfermeiros possuem oportunidades únicas para ajudar seus pacientes a deixarem de fumar. Os objetivos deste estudo foram identificar o consumo, as atitudes e os conhecimentos recebidos sobre o tabaco entre enfermeiras de um hospital. É um estudo descritivo, e foi realizado entre 204(48\%) enfermeiras do Hospital Arzobispo Loayza, em Lima, Peru, utilizando la Encuesta Mundial de Profesionales de la Salud (GHPS). Pelos resultados, pôde-se identifcar prevalência do uso na vida de tabaco, entre $67,1 \%$ da amostra, e no último mês $3 \%$. A maioria dos enfermeiros apresentou atitudes positivas e conhecem a importância e a responsabilidade de aconselhar os pacientes a parar de fumar. A maioria recebeu conhecimentos gerais sobre o tema, porém, a metade não recebeu conhecimentos específicos sobre o tratamento. Conclui-se, frente à importância do tema, que a presente pesquisa identificou a necessidade de mais estudos dessa natureza, bem como revisão curricular dos temas abordados a respeito do tema tabagismo.

Descritores: Tabagismo; Recursos Humanos de Enfermagem no Hospital; Enfermagem.

\section{Smoking Among Nurses of the National Hospital in Lima, Peru}

Nurses have unique opportunities to help their patients to stop smoking. The aim of this study was to identify, among nurses of a hospital, the consumption of smoking products, their attitudes and information received on smoking. It was a descriptive study, carried out with 204 (48\%) nurses of the Hospital Arzobispo Loayza, Lima, Peru. The Global Health Professional Survey (GHPS) was used. Among the sample the prevalence of smoking in life was $67.1 \%$ and $3 \%$ in the last month. The majority of nurses presented positive attitudes and knew the importance and responsibility of advising patients to quit. The majority had received general information regarding the topic, though half of them had not received specific information regarding treatment. In view of the importance of this theme, this study identified a need for further research of this nature as well as revision of the curricula regarding the approach toward the theme of smoking.

Descriptors: Smoking; Nursing Staff, Hospital; Nursing.

\section{Introducción}

El tabaquismo es uno de los comportamientos que pueden ser prevenidos, evitando así graves consecuencias para la salud, lo que es bien conocido por todos los profesionales de la salud. Este tema es considerado como un gran problema de salud pública y afecta cada vez más a las poblaciones jóvenes; anualmente, su consumo causa millones de muertes alrededor del mundo(1). La Organización Panamericana de la Salud (OPS) confirma estos datos y considera que las acciones para el control del uso de tabaco están entre las prioridades de las políticas sanitarias mundiales para las próximas décadas ${ }^{(2)}$.

Entre las estrategias para enfrentar el problema, los organismos internacionales consideran que los profesionales de la salud, como médicos, enfermeros y otros tienen un potencial enorme para desempeñar una función clave en la lucha contra el tabaco(3) y una gran responsabilidad en la prevención y tratamiento, debido a los conocimientos recibidos sobre el efecto del uso de tabaco durante su formación.

Por otro lado, en las últimas décadas, la literatura mundial ha presentado un número bastante considerable de estudios al respecto de la prevalencia del tabaquismo entre los enfermeros y estudiantes de enfermería, visualizando las preocupaciones sobre las implicancias de ese comportamiento en la salud de ese grupo y también en relación al papel que estos profesionales desarrollan en el ámbito de la prevención y de la promoción de 
la salud. Este hecho fue identificado en una revisión sistemática sobre consumo de tabaco entre enfermeros y estudiantes de enfermería, donde se señala que estos profesionales constituyen un gran grupo que promueven la salud; al mismo tiempo, los índices de consumo de tabaco de este grupo son considerablemente altos ${ }^{(4)}$, considerado por algunos autores como un hecho paradójico debido a que estando aptos en sus habilidades cognitivas y de interacción, los profesionales de la salud poseen la credibilidad y las oportunidades para ayudar a las personas a modificar su comportamiento con relación al hábito de fumar. Por tanto, el déficit de conocimiento, la falta de habilidades y la falta de programas educativos en los currículos de graduación acerca del tema pueden constituirse en barreras, para los enfermeros y otros profesionales de la salud, para implementar políticas de control y programas de intervención para el cese del tabaquismo; además el tabaquismo entre los profesionales de la salud constituye una influencia negativa sobre los pacientes que están intentando dejar de fumar. La falta de conocimiento formal sobre los riesgos del fumar, sobre el manejo de programas para el cese del consumo de cigarrillo y la falta de habilidades pueden ser vistas como las principales dificultades para que los enfermeros asuman sus funciones de educador frente al control del tabaquismo(5).

En el pasado, parece haber existido un consenso general, entre los estudios de prevalencia del consumo de tabaco entre los enfermeros en muchos países, de que el porcentaje de consumo de estos profesionales era equivalente al de las mujeres de la población, en general, siendo estas también muy elevadas. En Latinoamérica es sabido que cuanto más critica es la economía del país, más altos son los índices de consumo de tabaco en los diversos grupos de la población; también la práctica de desarrollar estudios, para monitorizar los índices de prevalencia del comportamiento de fumar, como en el caso de los enfermeros, aún son escasos.

Los estudios disponibles en la literatura demuestran índices de alrededor de 42,7\% en Chile(6), 43,1\% en México(7), 30\% en Brasil( ${ }^{(8)}$ y $22,1 \%$ en Argentina( ${ }^{(9)}$; a pesar de existir diferencias metodológicas y de tamaños de muestreo que no permiten comparaciones, se puede afirmar que los estudios se realizaron en enfermeras hospitalarias, que en su mayoría eran mujeres. En Perú, vienen siendo realizados esfuerzos considerables por los organismos gubernamentales para realizar investigaciones de forma más constante sobre el uso de diversos tipos de drogas, como medio de monitorizar la implementación de políticas públicas de control del uso.
Uno de los estudios recientes identificó que la prevalencia del uso del tabaco en profesionales de la salud es mayor que la prevalencia en la población general ${ }^{(10)}$.

De acuerdo al Centro de Información y Educación para la Prevención del Abuso de Drogas (CEDRO) 63,4\% de los peruanos ha fumado alguna vez en la vida; en esa encuesta se demostró que más de la mitad de la muestra eran consumidores actuales (último mes) y principalmente los usuarios eran adolescentes y jóvenes $^{(11)}$. Si consideramos los índices de la literatura, podemos afirmar que de cada diez enfermeros seis son fumadores.

Frente a la escasez de estudios acerca del tema y de la problemática presentada por el consumo de tabaco entre enfermeros, el presente estudio tuvo comos objetivos identificar la prevalencia y las actitudes del consumo de tabaco entre enfermeras hospitalarias.

\section{Método}

En el estudio se utilizó la metodología cuantitativa descriptiva. La población de estudio fue constituida por 425 (100\%) Licenciadas en Enfermería pertenecientes al Hospital Nacional Arzobispo Loayza en Lima, Perú. Los criterios de inclusión fueron trabajar como mínimo un año en el hospital y participar voluntariamente. Para el cálculo de la muestra se utilizó el porcentaje de uso tabaco en la vida de la población (60\%) y un error de 0,05, estimándose una muestra mínima de 195 entrevistados. Entretanto, la muestra fue constituida por 204 (48\%) enfermeros de diversos sectores del hospital: Medicina 52 (25,4\%), Consultorios Externos $42(20,5 \%)$, Maternidad $26(12,7 \%)$, Ginecología 25 (12,2\%), Cirugía 17 (8,3\%), Emergencia 15 (7,3\%), Pediatría 11 (5,3\%), Departamento de Enfermería 10 $(4,9 \%)$ y Sala de Operaciones 6 (2,9\%).

Para recolectar los datos, se utilizó el instrumento Encuesta Mundial de Profesionales de la Salud (GHPS)(12) previa autorización de CEDRO; este es un cuestionario autoadministrado con 49 preguntas, divididas en seis partes que considera preguntas relacionadas con: informaciones sociodemográficas, prevalencia de uso de tabaco entre profesionales de la salud, exposición al humo ambiental del tabaco, actitudes frente al tabaquismo, exposición al humo ambiental del tabaco, conductas frente al uso de tabaco y, entrenamiento o capacitación para trabajar con la cesación del tabaquismo. Como es la primera vez que se utiliza este instrumento entre enfermeras hospitalarias, fue necesario realizar la prueba de confiabilidad, obteniéndose un alfa de Cronbach de 0,7667. 
El proyecto fue aprobado por el Comité de Ética del Hospital Nacional Arzobispo Loayza. La encuesta se entregó a los enfermeros al inicio del turno y se recogió al término del mismo. La participación de las enfermeras fue voluntaria y anónima, garantizándose a los participantes la confidencialidad de las informaciones. Los participantes firmaron el Consentimiento Informado aceptando participar en el estudio. Los datos fueron analizados con el programa SPSS versión 13 y presentados en frecuencias y porcentajes de uso de tabaco.

\section{Resultados}

Entre las características sociodemográficas de los enfermeros se destacaron el género femenino 195
$(95,6 \%)$, con edades de 30 años o más $179(87,7 \%)$.

En cuanto a la prevalencia de vida del uso de tabaco, se identificó que $137(67,2 \%)$ enfermeros ya habían fumado una vez en la vida y en el consumo actual, apenas $6(3 \%)$ reportaron consumo en el último mes. De 132 (64,7\%) entrevistados, 107 (81\%) afirmaron que la edad en que probó por primera vez el tabaco fue cuando tenía más de 18 anos.

En relación a la exposición al humo ambiental, 18 participantes (10\%) estuvieron expuestos al humo dentro del hogar y 51 (25\%) fuera del hogar.

La Tabla 1 muestra que la mayoría de los enfermeros presentaron actitudes positivas para la función que tienen frente al tabaquismo.

Tabla 1 - Actitud y responsabilidad de los profesionales de salud frente a cesación del tabaquismo, según los enfermeros del HNAL. Lima - Perú, 2007

\begin{tabular}{|c|c|c|}
\hline & \multicolumn{2}{|c|}{$\mathrm{Si}$} \\
\hline & $\mathbf{n}$ & $\%$ \\
\hline Los profesionales de la salud deben ofrecer consejería sobre cesación de fumar a sus pacientes $(n=204)$ & 201 & 99,0 \\
\hline Se debería capacitar a los profesionales de salud sobre técnicas de cesación $(n=200)$ & 197 & 98,5 \\
\hline Los profesionales de salud tienen un rol a desempeñar en la consejería a sus pacientes sobre cesación de fumar ( $n=203$ ) & 198 & 97,5 \\
\hline Los Profesionales de la Salud son "modelos" para sus pacientes y público (n=201) & 191 & 95,0 \\
\hline Las posibilidades de dejar de fumar de un paciente aumentan cuando un profesional de salud le aconseja dejar de hacerlo $(n=200)$ & 185 & 92,5 \\
\hline Los profesionales de salud que fuman son menos propensos a orientar a sus pacientes sobre la cesación de fumar ( $n=202$ ) & 160 & 79,2 \\
\hline
\end{tabular}

La Tabla 2 presenta información acerca de capacitación acerca del tabaco, casi todas conocen los peligros del tabaco medioambiental o proveniente de otros fumantes $(91,2 \%)$ y la importancia de prevenir el inicio del consumo de tabaco en niños y jóvenes
$(86,85)$, sin embargo la proporción disminuye a la mitad en relación a la capacitación formal sobre la técnica de cesación $(55,2 \%)$ y en estrategias de control del tabaco en el país $(52,5 \%)$.

Tabla 2 - Conductas y entrenamientos a respecto del tabaquismo recibido por los enfermeros del HNAL durante la formación profesional. Lima - Perú, 2007

\begin{tabular}{|c|c|c|c|c|}
\hline & \multicolumn{2}{|c|}{ Si } & \multicolumn{2}{|c|}{ No } \\
\hline & $\mathbf{n}$ & $\%$ & $\mathbf{N}$ & $\%$ \\
\hline $\begin{array}{l}\text { ¿Se le ha explicado, en cualquiera de las clases, sobre los peligros para la salud provenientes de la exposición } \\
\text { al humo de tabaco ambiental o de segunda mano? }(n=203)\end{array}$ & 185 & 91,2 & 18 & 8,8 \\
\hline ¿Ha aprendido que es importante registrar en la historia clínica el uso de tabaco de un paciente? $(\mathrm{n}=203)$ & 180 & 88,2 & 23 & 11,8 \\
\hline ¿Es importante prevenir el inicio y el consumo de tabaco en niños, jóvenes y mujeres embarazadas? $(n=204)$ & 177 & 86,8 & 27 & 13,2 \\
\hline $\begin{array}{l}\text { ¿Ha aprendido sobre la importancia de brindar materiales educativos sobre cesación de fumar para ayudar a } \\
\text { pacientes que desean dejar de fumar? }(n=204)\end{array}$ & 150 & 73,5 & 54 & 26,5 \\
\hline ¿Ha recibido capacitación formal en técnicas para dejar de fumar para ser aplicadas en sus pacientes? $(\mathrm{n}=201)$ & 111 & 55,2 & 90 & 44,8 \\
\hline $\begin{array}{l}\text { ¿Ha participado en charlas, conferencias o talleres acerca de las estrategias de control del consumo de tabaco } \\
\text { implementadas en el Perú a nivel poblacional? }(n=204)\end{array}$ & 107 & 52,5 & 97 & 47,5 \\
\hline
\end{tabular}


En la Tabla 3 se muestra que la mitad de las participantes conocen las terapias de reemplazo de la nicotina para obtener la cesación $(62,5 \%)$ y el uso de antidepresivos en el tratamiento de cesación (52,5\%), mientras que un porcentaje menor conoce el Convenio Marco para el Control de Tabaco $(46,1 \%)$.

Tabla 3 - Contenidos recibidos sobre el tratamiento y control del tabaco durante el entrenamiento para enfermeras, Lima, Perú, 2007

\begin{tabular}{|c|c|c|c|c|}
\hline & \multicolumn{2}{|c|}{ Si } & \multicolumn{2}{|c|}{ No } \\
\hline & n & $\%$ & n & $\%$ \\
\hline Terapias de reemplazo de nicotina en programas de cesación de fumar (parches o chicles de nicotina) & 127 & 62,5 & 76 & 37,5 \\
\hline Uso de antidepresivos en los programas de cesación de fumar (como Bupropion o Zyban) & 107 & 52,5 & 97 & 47,5 \\
\hline El Convenio Marco para el Control del Tabaco & 94 & 46,1 & 110 & 53,9 \\
\hline
\end{tabular}

\section{Discusión}

La prevención del uso de tabaco y la promoción de cesación de ese consumo es una función importante de los enfermeros. Siendo el consumo de tabaco considerado como la mayor causa de muerte que es posible de prevenir, los profesionales de la salud tienen un potencial clave en la lucha contra la epidemia del tabaco(3), por lo tanto una estrategia, para reducir el número de daños y de consumo del tabaco, es incentivar la participación de los profesionales de la salud en la prevención y la consejería de cesación ${ }^{(3)}$.

En la presente investigación se identificó $67,2 \%$ de prevalencia de vida de uso del tabaco y $3 \%$ de uso actual, en una muestra compuesta en su mayoría por mujeres jóvenes, lo que es corroborado por la literatura internacional(4-10). De esa forma, parece que el comportamiento del fumar entre enfermeros puede estar ocurriendo de forma ocasional o esporádicamente, una vez que el consumo actual fue muy bajo. La literatura ofrece algunas explicaciones sobre los motivos que pueden estar contribuyendo para que esta categoría profesional fume en niveles comparados a la población general; estas explicaciones están relacionadas con aspectos sociales, culturales, estrés, además de la influencia de los propios compañeros de trabajo, las condiciones sociales y económicas, así como la educación ${ }^{(13)}$. Para obtener esas informaciones es necesario realizar otras investigaciones más especificas acerca del tema ya que 107 (81\%) enfermeros afirmaron que probaron por primera vez el cigarrillo a la edad de 18 años o más. Otra posible explicación sobre estos resultados sea la inserción de los jóvenes en la universidad, pues la mayoría de los estudiantes ingresa a los 18 años o más. Por otro lado, la prevalencia de vida de uso de cigarrillo entre los enfermeros puede no ser sorprendente, una vez que en el Perú los índices de uso de tabaco entre las mujeres es muy elevado(11).
El consumo de tabaco en las instalaciones del hospital por parte de los enfermeros fumadores fue muy poco, solo el 3\% fumaron en el ultimo mes, porcentaje muy bajo; entretanto más de la mitad ya fumaron en la vida. Ese hecho puede estar relacionado a las limitaciones del muestreo. Una vez que 52\% de la población-objetivo no participó de la investigación, deduciéndose la posibilidad de que la prevalencia fue subestimada debido al sesgo de respuesta, quiere decir que probablemente los fumantes quedaron fuera de la investigación.

Considerando los altos índices de prevalencia de vida del uso de cigarrillo en la población peruana en general y también entre los profesionales de salud, los enfermeros necesitan redoblar esfuerzos para obtener una mejor formación y capacitación sobre el tratamiento del tabaquismo y el cese del uso de cigarrillo.

\section{Actitudes y conocimientos hacia al tabaquismo}

La mayoría de los enfermeras poseen actitudes positivas en sus roles como profesionales de salud frente a este gran problema de salud pública y están de acuerdo en que tienen funciones importantes en la intervención de los problemas relacionados al uso de tabaco; sin embargo, manifiestan que recibieron contenidos muy generales respecto a este tema y sólo la mitad conoce las especificidades del tratamiento. Estos datos pueden ser indicadores de las fragilidades de los currículos de formación a nivel de pregrado, y que estos abordan poco sobre esa temática y/o lo hacen de manera general. La literatura internacional ha demostrado la eficacia de las acciones de los enfermeros en el tratamiento y cesación del tabaquismo. En ese sentido, se identificó que aunque la mayoría los enfermeros creen y aprueban sus responsabilidades para orientar a los pacientes en la cesación del tabaco, el porcentaje de enfermeros que relataron sus reales actuaciones fue muy pequeña(14). 
En el presente estudio casi todos los enfermeros afirmaron que los profesionales de la salud son modelos para sus pacientes (95\%), sin embargo la revisión de la literatura destaca que debido a la importancia atribuida a la función del enfermero como profesional de salud y la presión para actuar como buenos modelos, constituye un aspecto que es de fundamental importancia y debe ser investigado para entender los motivos que llevan a los enfermeros a fumar. En resumen, las experiencias, las percepciones y los comportamientos de los profesionales fumadores son reflejados en las mujeres, y personas jóvenes en general, lo que amerita realizar investigaciones sobre esos aspectos ${ }^{(13)}$

Los enfermeros aprueban que los profesionales de la salud deban aconsejar periódicamente a sus pacientes a dejar de fumar y también dejar de usar otros productos derivados del tabaco. Asimismo, mayoritariamente consideran que los profesionales de la salud tienen la función de brindar consejos e ofrecer información a sus pacientes sobre el cese del fumar $y$ que las oportunidades del paciente aumentan cuando un profesional de la salud les aconseja a dejar de fumar, Tabla 1. Estos resultados se encuentran corroborados en la literatura ${ }^{(4-9,13-14)}$, ya que cuanto más aptos y contando con actitudes positivas, estos profesionales tienen mayor posibilidad de abrir las ventanas de oportunidades para la implementación de intervenciones de prevención, e invitar a los pacientes a dejar el tabaquismo.

Estos datos están de acuerdo con los propuestos por la OPS que considera que los profesionales de la salud tienen un potencial enorme para desempeñar funciones claras en la lucha contra el tabaco(3) y una gran responsabilidad profesional y social en la prevención y tratamiento del cese al tabaco en la población; esto debido a los conocimientos que tienen sobre el efecto del uso de las drogas, recibido durante su formación. Asimismo, se considera que los profesionales de la salud pueden actuar contra el uso del tabaco de diversas formas: actuando como modelos de salud, brindando educación sanitaria, promoviendo la aplicación de la legislación sanitaria en establecimiento de salud y, sensibilizándolos sobre los problemas de salud derivados del tabaquismo. En el caso particular de enfermería, lo afirmado por las participantes coincide con la declaración del Consejo Internacional de Enfermeras acerca del rol ideal que tienen las enfermeras en la promoción de la cesación de tabaco; para esto es importante que estas profesionales tengan oportunidades para obtener el conocimiento necesario que las haga capaces de incentivar a otros a no iniciar el consumo de tabaco(15).

En relación al entrenamiento profesional acerca de tópicos sobre tabaco, la mayoría de los enfermeros destaca que conocen los efectos del humo de tabaco sobre la salud, la importancia de brindar material educativo para dejar de fumar y de registrar el consumo de tabaco en la historia clínica; sin embargo la proporción de conocimientos recibidos disminuye a medida que aumenta la especificidad del tratamiento, Tabla 2. La prevención de fumar y la promoción de actividades para motivar los pacientes a dejar de fumar es una función importante de los enfermeros.

En relación al conocimiento, se encontró déficit sobre las terapias de reemplazo de la nicotina, sobre el uso de antidepresivos para la cesación y sobre el Convenio Marco para el Control de Tabaco, Tabla 3. Este hecho viene ocurrido en las facultades y escuelas de enfermería peruana como también en otros países que destinan menos de dos horas a la enseñanza de temas sobre el tabaquismo. Estos resultados nos llevan a inferir que los temas discutidos en los currículos sobre el tabaco pueden estar destinados, en su mayor parte, a los efectos del tabaco en la salud. Hacemos énfasis en la necesidad de reforzar los contenidos específicos en las áreas clínicas, con técnicas de manejo para trabajar la cesación del tabaquismo.

Estos resultados nos llevan a aspectos identificados en la revisión de literatura, como la identificación de las necesidades de enseñanza, la aplicación de los métodos de enseñanza, la efectividad de los métodos de enseñanza en esta área, la verificación de los modelos curriculares para los profesionales de la salud sobre entrenamiento para el tratamiento del tabaquismo - los que todavía son pocos. En la revisión se demuestra que el incremento del método de enseñanza (por ejemplo la consejería basado en orientaciones a los pacientes, el juego de role playing y/o la combinación de varios métodos) son más efectivos para enseñar intervenciones sobre el tabaquismo que solamente las enseñanzas con métodos tradicionales. En conclusión estos métodos de enseñanza o sus combinaciones ya son usados en diversas áreas de la enfermería(15).

Los conocimientos sobre los efectos del tabaco probablemente hayan sido parte de temas transversales, tales como enfermedades pulmonares o cardiovasculares en donde el tabaco es mencionado; sin embargo es conocido que el tema del tabaquismo per se no es parte de todos los currículos de formación en el pregrado, con excepción de la Facultad de Enfermería de la Universidad Peruana Cayetano Heredia que viene desde 1999, reestructurando sus bases curriculares y entrenando a los docentes de enfermería en el ámbito del fenómeno de las drogas, con apoyo del Proyecto Interamericano: Formación y Capacitación de Enfermeras en Promoción de la Salud y Prevención del Uso Indebido de Drogas, 
promovido por la Comisión Interamericana para el Control del Abuso de Drogas (CICAD) ${ }^{(14)}$.

\section{Conclusión}

Ese estudio buscó identificar el consumo, las actitudes y los conocimientos sobre el tabaquismo, en una muestra de enfermeras que trabajaban en un hospital en Lima, Perú. Los estudios de esta naturaleza son importantes para conocer las necesidades reales de esta población y garantizar una efectiva actuación y formación profesional. De esta forma, la prevalencia de vida del uso de tabaco fue de $67 \%$; el uso actual tuvo un menor porcentaje, indicando un uso ocasional. Con unanimidad los enfermeros presentaron actitudes positivas frente a la responsabilidad profesional que deben asumir al enfrentarse al problema del consumo de tabaco, además poseen conocimientos generales al respecto del tema, pero sólo la mitad de la muestra conoce contenidos más específicos sobre el tratamiento para cesación del fumar. Estos profesionales poseen oportunidades únicas en su práctica para ayudar a las personas a cambiar los comportamientos relacionados al fumar, pero existe la necesidad de reforzar las técnicas de manejo y terapias utilizadas en el tratamiento del tabaquismo.

\section{Agradecimientos}

Agradecemos a la Comisión Interamericana para el Control del Abuso de Drogas/CICAD de la Secretaria de Seguridad Multidimensional/SSM de la Organización de los Estados Americanos/OEA, la Secretaria Nacional de Políticas sobre Drogas/SENAD do Gabinete de Seguridad Institucional/Brasil, la Escuela de Enfermería de Ribeirao Preto de la Universidad de Sao Paulo y Centro Colaborador de la Organización Mundial de la Salud para el Desarrollo de la Investigación en Enfermería, la población representada en los estudios de investigación, bien como a las autoridades de las universidades representadas por los participantes del Programa En-Line de Especialización en Investigación sobre el Fenómeno de las Drogas - PREINVEST, periodos 2005, 2006, 2007 y 2008.

\section{Referencias}

1. Jha P, Chaloupka F, Moore J, Gajalakshmi V, Gupta P, Peck R, et al. Tobacco addiction. In: Jamison DT, Breman J, Measham A, Galleyne M, Evans D, Jha P, Mills A, et al. Disease control priorities in developing countries. $2^{\mathrm{a}}$ ed; . Washington: Oxford University Press and World Bank; 2006. 869-86.

2. Organización Panamericana de la Salud. Tabaco o Salud. Situación en las Américas. Washington DC: OPS; 2001. Publicación Científica No 536.

3. Organización Mundial de la Salud/Organización Panamericana de la Salud - OMS/OPS. La función de los profesionales de la salud en el control del tabaco. Ginebra: OMS; 2004.

4. Smith DR. A systematic review of tobacco smoking among nursing students. Nurse Educ Practice. 2007; (7):293-302.

5. Rower K, Clark JM. Why nurses smoke: a review of the literature. Int J Nurs Studies. 2000; (37):173-81.

6. Osorio XS, Rivas ER, Jara JB. Prevalencia de tabaquismo en enfermeras de la IX Región, Chile. Rev Med Chile. Mar. 2003;131(3):269-74.

7. Arenas L, Jasso R, Hernández I, Martinez $P$, Menjivar A. Prevalencia de tabaquismo de médicos y enfermeras en los estados de Morelos y Guanajuato. Rev Inst Nal Enf Resp Mex. 2004; 17(4):261-5.

8. Pillon SC, Dunn J Laranjeira R. O comportamento do fumar entre enfermeiras. Acta Paul Enferm. 2002; 15(2):65-70

9. Ferrero F, Castaños C, Durán P, Blenguini MT. Grupo de Estudio del Tabaquismo en la Residencia de Pediatría. Prevalencia del consumo de tabaco en médicos residentes de pediatría en Argentina. Rev Panam Salud Publica. 2004; 15(6):395-9.

10. Chanamé E, Matta M, Marticorena L, Zárate M, Zavaleta A. Prácticas sobre consumo de tabaco y otras drogas en estudiantes de postgrado de la Facultad de Enfermería de la Universidad Peruana Cayetano Heredia. Monografía de Investigación n.22. Lima: Centro de información y Educación para la Prevención del Abuso de Drogas; 2002.

11. Zavaleta A, Castro De La Matta R. Epidemiología de las drogas en la población urbana peruana, 2003. [Monografía de Investigación 23.] Lima: Centro de Información y Educación para la prevención contra el abuso de drogas (CEDRO); 2004.

12. CEDRO; OPS/OMS. Encuesta Mundial de Profesionales de la Salud (GHPSS). Centro de Información y Educación para la prevención contra el abuso de drogas CEDRO. Lima, 2007.

13. Whyte F, Kearney N. Enhancing the nurse's role in tobacco control. En Tobacco Control Factsheets.

[aceso en: 16 Octubre 2006]. [6 pantallas]. Disponible en: http://factsheets.globalink.org/en/nursesrole.shtml

14. Comisión Interamericana para el Control del Abuso de Drogas - CICAD. Proyecto de la CICAD de las Escuelas de Enfermería sobre Prevención del Uso y Abuso de Drogas, Integración Social y Promoción de la Salud en América Latina. Washington; [aceso en: 8 febrero 2008] [6 pantallas] Disponible en: http://www. cicad.oas.org/Reduccion_Demanda/esp/Enfermeras/Peru/ PeruIII.htm

15. Wewers ME, Kidd K, Armbruster D, Sarna L. Tobacco dependence curricula in U.S baccalaureate and graduate nursing education. Nurs Outlook. 2004; (52):95-101.

Recibido: 6.3 .2009

Aceptado: 6.4.2009 